
Revue
de Ihistoire des religions
Revue de l'histoire des religions
$4 \mid 2016$
Fides ex auditu. Théologie et audition dans le christianisme médiéval en Occident

\title{
Combattre « la vaine volupté de l'oreille » dans la rhétorique cistercienne
}

Fighting the "Vain Pleasure of the Ear » in Twelfth-Century Cistercian Rhetoric

\section{Marie Formarier}

\section{OpenEdition}

\section{Journals}

Édition électronique

URL : http://journals.openedition.org/rhr/8646

DOI : $10.4000 /$ rhr.8646

ISSN : 2105-2573

Éditeur

Armand Colin

Édition imprimée

Date de publication : 15 décembre 2016

Pagination : 533-555

ISBN : 978-2-200-93062-2

ISSN : 0035-1423

Référence électronique

Marie Formarier, « Combattre « la vaine volupté de l'oreille » dans la rhétorique cistercienne », Revue de I'histoire des religions [En ligne], 4 | 2016, mis en ligne le 15 décembre 2019, consulté le 23 janvier 2021. URL : http://journals.openedition.org/rhr/8646 ; DOI : https://doi.org/10.4000/rhr.8646 


\section{Combattre « la vaine volupté de l'oreille» dans la rhétorique cistercienne}

Cet article propose une analyse stylistique et musicale des outils rhétoriques mis en ouvre par deux auteurs cisterciens souhaitant dénoncer le plaisir excessif procuré par l'écoute musicale dans certaines circonstances. Le premier texte étudié est le chapitre XXIII du livre II du Miroir de Charité d'Aelred de Rievaulx. Son examen minutieux montre l'influence indéniable de la rhétorique antique du blâme, afin de dénoncer une pratique nouvelle : la polyphonie vocale. Ensuite, l'attention se porte sur différents récits exemplaires écrits par Conrad d'Eberbach en 1190 dans le Grand Exorde. L'ensemble de ce corpus a pour objectif de remettre en cause des pratiques musicales contraires à la dévotion cistercienne, mais aussi d'affirmer, concrètement, la légitimité de la rhétorique dans le cloître.

\section{Fighting the « Vain Pleasure of the Ear » in Twelfth-Century Cistercian Rhetoric}

This paper offers a stylistic and musical analysis of the rhetorical tools employed by two Cistercian authors who wish to denounce the excessive pleasure caused by listening to music in certain circumstances. The first text is Chapter 23 of Book II of the Mirror of Charity, written by Aelred of Rievaulx. Careful analysis shows the undeniable influence of the ancient rhetoric of blame, applied to a new practice: vocal polyphony. I then focus on different stories written by Conrad of Eberbach in 1190 in his Grand Exorde. This corpus aims, in different ways, to criticize musical practices contrary to Cistercian devotion and also to defend, in practice, the legitimacy of rhetoric in the cloister. 


\section{INTRODUCTION}

Si l'on admet à la suite de Wim Verbaal que «ce qu'on peut retrouver chez les écrivains $\mathrm{du} \mathrm{XI}^{\mathrm{e}}$ et $\mathrm{XII}^{\mathrm{e}}$ siècle constitue en grande partie l'héritage classique dont on dispose aujourd'hui ${ }^{1}$, on reconnaîtra sans peine que la tâche du philologue qui voudrait évaluer précisément l'influence au Moyen Âge de la culture antique est immense et complexe. Les travaux menés par Birger Munk Olsen sur cette question épineuse sont un outil indispensable, mais révèlent aussi les lacunes documentaires auxquelles nous sommes confrontés. Ainsi, le cas des Cisterciens, qui m'intéresse, s'avère problématique. Sur seize inventaires de bibliothèques cisterciennes, sept, parmi lesquels celui de Clairvaux, ne mentionnent pas d'œuvres classiques $^{2}$. Le problème se corse lorsqu'il s'agit de se pencher plus précisément sur l'héritage de la rhétorique antique. Certes, l'inventaire de la bibliothèque de Rievaulx mentionne, parmi les classiques, une Rhetorica ${ }^{3}$ qui pourrait bien être un manuscrit du De inventione ou de la Rhetorica ad Herennium, les deux traités les plus largement diffusés au Moyen Âge ${ }^{4}$. Mais nous n'en avons aucune preuve. À vrai dire, l'influence des classiques chez les Cisterciens doit être davantage appréhendée comme un contexte littéraire et esthétique plus général, caractéristique du XII siècle ;

1. Wim Verbaal, «Teste Quintiliano. Jean de Salisbury et Quintilien», Quintilien ancien et moderne, dir. Perrine Galand et al., Turnhout, Brepols, 2010, p. $155-170$ (p. 158)

2. Voir Birger Munk Olsen, L'étude des auteurs classiques latins aux XI et XII ${ }^{e}$ siècles, t. IV, $2^{\mathrm{e}}$ partie, Paris, CNRS éditions, 2014, p. 404.

3. Voir Anne-Marie Turcan-Verkerk, «To What Extent Were the TwelfthCentury Cistercians Interested in Rhetorical Treatises ? », The Art of Cistercian Persuasion in the Middle Ages and Beyond. Caesarius of Heisterbach's Dialogue on Miracles and its Reception, dir. Victoria Smirnova et al., Leyde, Brill, 2015, p. 51-78 (p. 54). B. Munk Olsen, L'étude des auteurs classiques..., t. III, $1^{\mathrm{e}}$ partie, Paris, CNRS éditions, 1987, p. 208.

4. James J. Murphy, Latin Rhetoric and Education in the Middle Ages and Renaissance, Alderhot, Ashgate, 2005, chap. I, p. 1-10 ; chap. XII, p. 200-201. Sur le cas de Cicéron en général aux $\mathrm{XI}^{\mathrm{e}}$ et $\mathrm{XII}^{\mathrm{e}}$ siècles, voir $\mathrm{B}$. Munk Olsen, t. IV, $2^{\mathrm{e}}$ partie, p. 420-421. Concernant les manuscrits du De Inventione et de la Rhetorica ad Herennium, voir B. Munk Olsen, L'étude des auteurs classiques..., t. IV, $1^{\text {e }}$ partie, Paris, CNRS éditions, 2009, p. 297-299. Des manuscrits integri sont attestés à partir de la fin du $\mathrm{x}^{\mathrm{e}}$ siècle. 
de plus, l'analyse de cet héritage n'est opérationnelle qu'à travers l'examen minutieux des textes, si l'on admet avec Étienne Gilson que «nourris de Cicéron et de saint Augustin, ils (les Cisterciens) ont renoncé à tout sauf à l'art de bien écrire $»^{5}$.

L'enseignement de Cicéron était ainsi connu principalement à travers le De inventione et la Rhetorica ad Herennium qui lui était attribuée, mais aussi indirectement, par le biais de florilèges ou d'abrégés, ou bien encore à travers la lecture qui en était offerte dans l'Antiquité tardive, notamment par Augustin dans le livre IV du De doctrina christiana ${ }^{6}$, dans les commentaires du grammairien Marius Victorinus (milieu du IV siècle) ou bien encore dans ceux de Grillius ( $\mathrm{v}^{\mathrm{e}}$ siècle). Plusieurs générations de commentaires médiévaux se succèdent ensuite: Birger Munk Olsen dénombre en effet neuf commentaires différents du De inventione, cinq de la Rhetorica ad Herennium, tous pourvus d'introduction ${ }^{7}$. Par ailleurs, l'Institution oratoire de Quintilien, constitue l'une des bases de l'enseignement monastique du trivium $^{8}$. Le texte s'est transmis par le biais de fragments et de résumés fournis par la tradition grammairienne du $\mathrm{IV}^{\mathrm{e}}$ siècle, mais il a aussi pu circuler en version intégrale, notamment dans le milieu anglo-normand comme en témoignent le Metalogicon et le Policraticus de Jean de Salisbury9. Enfin, comme l'ont montré les recherches menées par Anne-Marie Turcan-Verkerk, le XIII ${ }^{\mathrm{e}}$ siècle voit aussi fleurir de nouveaux traités de la parole (ars dictaminis, ars poetriae, ars praedicandi) ${ }^{10}$.

C'est en tenant compte de ce foisonnement culturel du XII siècle et de cet usage répandu, mais souvent masqué, des sources antiques dans les textes monastiques, en particulier cisterciens, que je

5. Étienne Gilson, La théologie mystique de saint Bernard, Paris, Vrin, 1934, p. 19.

6. J. J. Murphy, Latin Rhetoric..., chap. I, p. 5.

7. B. Munk Olsen, t. Iv, $1^{\mathrm{e}}$ partie, p. 62-67.

8. Damien Boquet, L'ordre de l'affect au Moyen Âge. Autour de l'anthropologie affective d'Aelred de Rievaulx, Caen, Publications du CRAHM, 2005 , p. 37.

9. J. J. Murphy, Latin Rhetoric..., chap. I, p. 4 ; W. Verbaal, «Teste Quintiliano... », p. 162. Sur la culture classique de Jean de Salisbury, voir B. Munk Olsen, L'étude des auteurs classiques..., t. IV, $2^{\mathrm{e}}$ partie, p. 419. Sur la réception de Cicéron par Jean de Salisbury, voir ibid. p. 425. Sur le fait qu'il ait détenu personnellement une copie de l'Institution Oratoire, voir ibid. p. 435.

10. Une synthèse de ces travaux est présentée dans l'article cité, «To What Extent... ?». 
souhaiterais m'attacher précisément au cas d'Aelred de Rievaulx et à son influence dans les exempla cisterciens de la fin du XII ${ }^{\mathrm{e}}$ siècle, notamment dans le Grand Exorde, un recueil composé par Conrad d'Eberbach. Je considère ces écrits cisterciens comme relevant de la rhétorique, dans le sens où l'énoncé cherche à agir sur le lecteur/ auditeur, à modifier son comportement, à le persuader de la nécessité de respecter les normes cultuelles et spirituelles défendues. Cette production discursive et narrative a bien «le pouvoir d'édifier des représentations capables d'agir sur le public et d'emporter la conviction $»^{11}$. L'efficacité persuasive de l'énoncé, liée avant tout à la réussite interactionnelle de la prestation discursive, autrement dit à la manière dont le locuteur parvient à inspirer la confiance de son interlocuteur et à le persuader d'adopter un point de vue, une croyance ou un comportement, reste difficile à évaluer pour une production désormais figée par l'écrit. Néanmoins, l'analyse multimodale du discours permet d'en entrevoir certains aspects. Comme le souligne Ruth Amossy, «les caractéristiques psychologiques et physiques ne sont pas données immédiatement et doivent de ce fait être reconstruites par le lecteur. C'est à travers le niveau de langue, le choix des mots, l'usage des expressions toutes faites, le rythme, l'humour, etc., que le lecteur va imaginer la voix et le corps de celui qui lui parle sans qu'il puisse l'entendre ou le voir concrètement $»^{12}$. C'est dans cette perspective que je proposerai dans cet article l'analyse de plusieurs extraits de cette production cistercienne : il s'agit avant tout d'en évaluer, autant que possible, la force d'action sur l'auditoire.

Lorsqu'il relate sa rencontre avec l'œuvre de Cicéron, Aelred souligne la qualité de la démonstration menée par l'Arpinate, mais aussi la douceur de son éloquence ${ }^{13}$. Sa connaissance du De Amicitia, et plus largement de la culture cicéronienne, nourrit de toute évidence les réflexions qu'il mène dans le Miroir de la Charité composé en $1142^{14}$. Cette influence de la culture oratoire antique se mesure

11. Ruth Amossy, La présentation de soi. Ethos et identité verbale, Paris, Presses Universitaires de France, 2010, p. 19.

12. R. Amossy, La présentation de soi..., p. 36.

13. B. Munk Olsen, L'étude des auteurs classiques..., t. Iv, $2^{\mathrm{e}}$ partie, p. 416 : «Tandem aliquando mihi venit in manus liber ille quem de amicitia Tullius scripsit ; qui statitm mihi et sententiarum gravitate utilise, et eloquentie suavitate dulcis apparebat »(prologue du De Spirituali amicitia).

14. Sur cette influence, voir D. Boquet, L'ordre de l'affect..., p. 40-46. 
autant par les thématiques et les réminiscences lexicales que par un souci constant apporté au style (figures de pensée, jeux énonciatifs, jeux phoniques et rythmiques). C'est la raison pour laquelle je souhaiterais appréhender cette influence comme un matériau à la fois conceptuel et stylistique, remodelé au service d'une cause nouvelle : la critique virulente de l'usage d'instruments de musique et de la polyphonie dans la liturgie et, en regard, l'apologie des bonnes règles d'écoute. Suivant la célèbre démonstration augustinienne consacrée au chant liturgique, Aelred préconise un dispositif de prière et d'écoute légitime et constructif. Légitime car il s'inscrit dans un cadre très contraint, qui sonne comme un écho à certains textes antiques sur lesquels je vais revenir. Constructif puisqu'il constitue un préalable à la méditation monastique, exercice pour lequel Aelred s'affirme comme un pédagogue de premier choix, comme en témoigne le traité sur La Vie de recluse écrit plus tard, en 1160. L'expérience sensorielle et émotionnelle est donc le sujet principal du chapitre XXIII du deuxième livre du Miroir de la Charité, consacré aux « vaines voluptés de l'oreille ». Il s'agit pour Aelred de fournir le modus operandi de l'écoute : que faut-il écouter ? Comment faut-il écouter? Pour quoi faire ? Or ces règles d'écoute deviennent ensuite dans la littérature exemplaire cistercienne un sujet récurrent, traité selon un dispositif différent que j'étudierai.

Le recueil d'exempla intitulé Grand Exorde et rédigé par Conrad d'Eberbach autour de 1190 reprend à son compte la question de l'écoute et du chant. Document d'archive, au même titre que le Petit Exorde compilé dans les débuts de l'Ordre, ce recueil est aussi écrit dans un contexte polémique : il entend répondre aux critiques émanant des milieux clunisiens, mais également de l'Ordre lui-même, qui connaît plusieurs révoltes de convers. C'est dans cette perspective que la volupté de l'oreille est évoquée et mise en scène à travers plusieurs exempla. Néanmoins, ce plaisir excessif de l'écoute provient moins du public présent que du chanteur lui-même, qui admire sa propre prestation et se retrouve ainsi coupable de vanité. Cette mise en scène d'une volupté suscitée par sa propre virtuosité vocale s'accompagne d'une mise en garde : ce comportement, indigne d'un moine, est contraire à la dévotion authentique et risque fort de mener celui qui s'y risque aux supplices de l'Enfer, dont la vision effroyable s'impose au lecteur saisi de crainte. Ainsi, au lieu d'adopter une perspective 
prescriptive, le narrateur de l'exemplum emprunte les détours de la narration afin de mettre en scène les comportements à louer ou blâmer. L'influence d'Aelred peut ainsi être évaluée selon deux paramètres : la permanence éventuelle d'un contenu doctrinal ainsi que des procédés oratoires (choix lexicaux, outils stylistiques du blâme et de l'éloge, musicalité de la langue).

Que ce soit dans l'œuvre d'Aelred comme dans celle de Conrad, la présentation des bonnes règles de l'écoute s'inscrit bien dans une perspective rhétorique, autrement dit dans un "discours qui cherche à être efficace dans la mesure où il prétend agir sur les états mentaux d'un auditoire $»^{15}$, voire sur sa manière d'être et son comportement pratique. En d'autres termes, l'objectif poursuivi par ces textes est d'agir sur la foi des auditeurs, appréhendée autant comme une croyance que comme un ensemble de gestes qui la matérialisent et la signifient aux yeux de tous.

Par conséquent, l'enjeu de mon travail est multiple : comprendre comment les Cisterciens, sur l'impulsion de Bernard de Clairvaux, se saisissent des normes antiques de l'écoute ${ }^{16}$, afin de se les approprier et de les adapter à la dévotion qui est la leur ; évaluer l'évolution de ces normes, mais aussi des modalités de leur transmission, en interrogeant l'influence des difficultés structurelles et spirituelles que connaît l'Ordre à la fin du XII ${ }^{\mathrm{e}}$ siècle, notamment au moment de la rédaction du Grand Exorde.

\section{BLÂMer les MAUVAISES PRATIQUES MUSICALES DANS le Miroir de Charité}

Plusieurs recherches ont d'ores et déjà été menées sur le chapitre XXIII du deuxième livre du Miroir de Charité d'Aelred, notamment par Bruno W. Holsinger ${ }^{17}$ ou encore Emma Dillon ${ }^{18}$.

15. Emmanuelle Danblon, «La construction de l'autorité en rhétorique », Semen, 2006, p. 2 (http://semen.revues.org/1983).

16. Une amorce de ce travail est menée dans Marie Formarier, «La perception intuitive chez les Cisterciens: quels modèles antiques ?», Pallas, 98, 2015, p. 183-196.

17. Bruno W. Holsinger, Music, Body, and Desire in Medieval Culture: Hildegard of Bingen to Chaucer, Stanford, Stanford University Press, 2001, p. 73.

18. Emma Dillon, «Representing Obscene Sound», Medieval Obscenities, dir. Nicola Mc Donald, Centre of Medieval Studies, University of York, Boydell $\&$ Brewer, 2006, p. 55-84. 
S'inscrivant dans une perspective musicologique, elles mettent l'accent sur le rapport entre polyphonie et obscénité, mais ne tiennent pas compte de la qualité rhétorique de la démonstration, ni du rapport que ce texte d'Aelred peut entretenir avec d'autres textes antiques ou bien avec les écrits de Bernard de Clairvaux, ni des enjeux spirituels qu'il pose. Or, le problème de l'expérience de Dieu et le rôle des émotions dans la croyance et la méditation, étudiés selon des angles d'approche différents par Olivier Boulnois ${ }^{19}$ et Damien Boquet ${ }^{20}$, sont au cœur de la démonstration de ce chapitre car «l'enseignement de saint Bernard et d'Aelred de Rievaulx cherche rarement à résoudre d'épineuses questions théologiques, mais plutôt à expliquer à quelles conditions la rencontre de Dieu et de l'homme est possible $\gg^{21}$.

En effet, l'objectif d'Aelred est de défendre « une théologie vécue, une théologie pratique, qui englobe toute l'existence et s'appuie sur l'expérience $»^{22}$. Ainsi, selon les termes de Charles Dumont, éditeur du Miroir de la Charité, «il s'agit ici de contempler simplement la scène évangélique en elle-même et pour elle-même, de se la représenter, de s'y rendre présent, de prendre part au drame, ou mieux, d'être pris par le dialogue ou l'émotion du moment $»^{23}$. En d'autres termes, la lecture du texte biblique pour Aelred doit inciter le moine à se représenter la scène mentalement et à y participer ${ }^{24}$. De cet exercice de visualisation naît la méditation proprement dite, par laquelle le moine s'immobilise hors du temps et de l'espace et se détache des contingences matérielles, afin de trouver un état propice à ce qu'Augustin appelle la «vision spirituelle $»^{25}$, une vision qui ne prend appui sur aucune donnée sensorielle immédiate mais sur le matériau de l'esprit et de la mémoire. Dès lors, comment Aelred parvient-il à mobiliser cette théologie pratique au service de la pédagogie de l'écoute?

19. Olivier Boulnois, Au-delà de l'image. Une archéologie du visuel au Moyen Âge Ve-XVe siècle, Paris, Seuil, 2008.

20. D. Boquet, L'ordre de l'affect au Moyen Âge...

21. O. Boulnois, Préface, dans Philippe Nouzille, Expérience de Dieu et théologie monastique au XII siècle, Paris, Cerf, 1999, p. 12.

22. O. Boulnois, Préface..., p. 13.

23. Charles Dumont, «Introduction », Aelred de Rievaulx, La vie de recluse, p. 26-27, cité par Ph. Nouzille, Expérience de Dieu..., p. 138.

24. Ph. Nouzille, Expérience de Dieu..., p. 137.

25. Augustin, De Genesi ad Litteram, XXIV, 51. 
Le tableau qu'Aelred dresse au début du chapitre s'inscrit à plusieurs titres dans la tradition antique. En premier lieu, il admet implicitement les normes comportementales et vocales édictées dans la rhétorique, notamment dans la Rhétorique à Hérennius, largement diffusée au Moyen Âge. En effet, émettre des «vociférations» féminines était indigne d'un orateur parce que l'ethos viril qu'il devait adopter aurait alors été mis en danger ${ }^{26}$. Par ailleurs, le discours tenu par Aelred fait écho aux critiques virulentes jadis adressées dans l'Antiquité à l'encontre de certains orateurs jugés comme efféminés en raison de leurs intonations vocales et de leurs gestes, inspirés de la pratique théâtrale ${ }^{27}$. Aussi l'élève de rhétorique chez Quintilien devait-il adopter une voix virile dans la lecture ${ }^{28}$ et se détourner de la musique «jouée désormais sur scène, efféminée et affaiblie par des modes obscènes $»^{29}$. Cette décadence était due, selon Quintilien, à la volonté des orateurs de se plier au mauvais goût du peuple ${ }^{30}$. Par conséquent, la force virile (virilis robor chez Quintilien, virilis vigor chez Aelred) se caractérise par le maintien d'un équilibre entre deux extrêmes : ni trop rude, ni trop mou. Elle ne doit être pas être brisée (fracta), amollie ou pervertie par une gestuelle excessive. Enfin, la critique du théâtre était également un leitmotiv dans la littérature tardive. Tertullien blâmait le caractère efféminé de l'acteur de pantomime ${ }^{31}$, tandis qu'Augustin mettait en garde son lecteur contre les émotions excessives que ce genre de spectacle procure ${ }^{32}$, raillant la vénalité des acteurs et la versatilité du public $^{33}$. Ainsi, lorsqu'il déplore le manque de virilité des chanteurs de polyphonie, Aelred s'inscrit dans une longue tradition selon laquelle la parole publique se doit de garantir le maintien d'un ethos digne d'un homme honnête, ni acteur, ni efféminé :

Vnde, quaeso, cessantibus iam typis et figuris, unde in ecclesia tot organa, tot cymbala? Ad quid, rogo, terribilis ille follium flatus,

26. Rhétorique à Hérennius, III, 22.

27. Marie Formarier, «Discours et ethos viril chez Cicéron et Quintilien », Le rapport éthique au discours, dir. Charles Guérin et al., Bern, P. Lang, 2013, p. 177-198.

28. Quintilien, Institution Oratoire, I, 8, 2.

29. Quintilien, Institution Oratoire, I, 10, 31.

30. Quintilien, Institution Oratoire, II, 12, 10.

31. Tertullien, Les spectacles, 17.

32. Augustin, Confessions, II, 2.

33. Augustin, La musique, I, 6, 11-12. 
tonitrui potius fragorem, quam vocis exprimens suavitatem ? Ad quid illa vocis contractio et infractio ? Hic succinit, ille discinit, alter supercinit, alter medias quasdam notas dividit et incidit. Nunc vox stringitur, nunc frangitur, nunc impingitur, nunc diffusiori sonitu dilatatur. Aliquando, quod pudet dicere, in equinos hinnitus cogitur, aliquando virili vigore deposito in femineae vocis gracilitates acuitur, nonnunquam artificiosa quadam circumvolutione torquetur et retorquetur. Videas aliquando hominem aperto ore quasi intercluso halitu exspirare, non cantare, ac ridiculosa quadam vocis interceptione quasi minitari silentium; nunc agones morientium, vel exstasim patientium imitari ${ }^{34}$.

Comme le souligne Bruno W. Holsinger ${ }^{35}$, ce passage est appréhendé avec beaucoup de circonspection par les musicologues, qui n'y voient pas une description fiable de pratiques musicales, mais plutôt une diatribe véhémente contre un désordre moral, lié à la polyphonie. Le caractère obscène de celle-ci pourrait être lié à l'usage de certains modes jugés comme lascifs, notamment dans la théorie de Boèce, ou plus simplement à la présence de voix aiguës, rendue nécessaire par l'ambitus mélodique ${ }^{36}$. Quoi qu'il en soit, ce

34. Aelred de Rievaulx, De Speculo Caritatis, II, 23, 67: «Pourquoi, je le demande, figures et symboles ayant disparu, pourquoi dans l'église tant d'orgues, tant de cymbales ? Pourquoi, s'il vous plaît, le vent effrayant de ces soufflets plus évocateurs du fracas du tonnerre que de la douceur de la voix ? Pourquoi ces voix qui se contractent et se brisent ? L'un chante en-dessous des autres, un autre à côté, un troisième au-dessus, un autre encore divise certaines notes et les interrompt en plein milieu. La voix tantôt s'étrangle et tantôt se brise, tantôt elle éclate et tantôt elle s'amplifie en larges sonorités. C'est honteux à dire, mais elle est parfois forcée au point de devenir hennissement de cheval, et parfois, perdant toute vigueur masculine, elle s'amenuise en voix féminine, grêle et fluette. Il n'est pas rare qu'elle se torde ou se détorde en d'artificielles circonvolutions. On voit parfois quelqu'un expirer l'air la bouche ouverte comme s'il avait le souffle coupé : il ne chante plus mais c'est comme s'il voulait faire peur au silence par une ridicule syncope de la voix, ou bien imiter l'agonie des mourants ou les soupirs des grands malades ». Trad. Ch. Dumont.

35. B. W. Holsinger, Music, Body and Desire..., p. 160-161. Voir également E. Dillon, « Representing Obscene Sound », p. 80.

36. Bonnie J. Blackburn, « The lascivious career of B flat », Eroticism in Early Modern Music, dir. B. J. Blackburn et Laurie Stras, Burlington, Ashgate, 2015, p. 19-42 (p. 29-30). E. Dillon («Representing Obscene Sound», p. 80) émet également l'hypothèse que certaines techniques vocales comme le vibrato peuvent produire, aux oreilles d'Aereld, ce caractère obscène. Elle souligne néanmoins qu'il existe bien peu de preuves sur les manuscrits. Enfin, B. W. Holsinger (Music, Body and Desire..., p. 159) propose d'établir une corrélation entre cette critique de la polyphonie et une polémique concernant la sodomie au XII ${ }^{\mathrm{e}}$ siècle. Cette analyse repose, me semble-t-il, sur une interprétation biaisée des textes, mais elle suggère au moins une piste de réflexion. 
passage fait appel aux outils rhétoriques de l'éloquence du blâme. La présence de l'instance énonciative est extrêmement marquée, dans les incises (quaeso, rogo, quod pudet dicere) et dans les comparaisons (potius... quam, quasi intercluso halitu, quasi). Il ne s'agit plus d'énoncer ou de conseiller, mais bien de provoquer des émotions (honte, crainte, répulsion, colère) et d'espérer, ainsi, une prise de conscience de la part du lecteur ainsi qu'une évolution, le cas échéant, du comportement monastique.

Cette posture en surplomb permet à Aelred d'émettre un jugement défavorable et de mettre en garde le lecteur contre de tels procédés. L'énoncé est ainsi ponctué par la répétition de marqueurs syntaxiques (unde, tot, ad quid, nunc, aliquando) qui permettent de dessiner les contours de ce portrait à charge. Le regard se pose tour à tour sur les chanteurs (hic... ille... alter... alter), selon un procédé qui a fait ses preuves dans l'art antique du portrait ${ }^{37}$. Les polyptotes (succinit, discinit, supercinit; torquetur et retorquetur) et les homéotéleutes (contractio et infractio ; stringitur, frangitur, impingitur) ou encore le chiasme final (minitári siléntium... patiéntium imitári), renforcé par des cadences accentuelles (cursus tardus et cursus velox), permettent de distinguer la cible de cette critique acerbe: les circonvolutions du chant polyphonique, pareilles aux déhanchements obscènes des histrions, déjà évoqués par Quintilien : «Interim histrionicis quibusdam gestibus totum corpus agitatur, torquentur labia, rotant oculi, ludunt humeri, et ad singulas quasque notas digitorum flexus respondet. Et haec ridiculosa dissolutio vocatur religio ; et ubi haec frequentius agitantur, ibi deo honorabilius serviri clamatur $»^{38}$. Aux torsions des mélodies polyphoniques répondent les ondulations de tout le $\operatorname{corps}^{39}$, dans une chorégraphie qui rappelle davantage les acteurs de pantomime

37. Par exemple chez Ovide, dans le livre III de l'Ars Amatoria, notamment aux v. 283-285: le poète termine son énumération en attirant les regards sur la belle dont le rire déforme le visage, puis sur celle qui rit comme une ânesse...

38. Aelred de Rievaulx, De Speculo Caritatis, II, 23, 67 : «Pendant ce temps, tout le corps s'agite en gestes de comédiens, les lèvres se tordent, on roule les yeux, on joue des épaules, tandis qu'à chacune des notes répond une flexion des doigts. Et l'on appelle religion ce grotesque relâchement ! Et l'on proclame que là où ces choses se reproduisent plus fréquemment, là aussi Dieu est plus honorablement servi!»

39. Concernant l'attention portée par Aelred sur le spectacle visuel autant qu'auditif offert par la polyphonie, voir E. Dillon, « Representing Obscene Sound », p. 80. 
que les orateurs du forum. La battue de la pulsation (digitorum flexus) est un trait caractéristique du musicien, mais un travers dans la rhétorique ${ }^{40}:$ le discours est alors soumis à une mélodie et adopte une logique strictement musicale qui tend à diluer le sens. Le travestissement ne se cantonne donc pas aux apparences : il touche l'exercice de la prière, ainsi que le discours tenu sur celle-ci. Le renversement des valeurs est clairement exprimé dans la dernière phrase de cet extrait, charpentée par une structure syntaxique et rythmique appuyée. Outre les marqueurs corrélatifs ( $u b i \ldots i b i)$ et les homéotéleutes (frequentius... honorabilius, agitantur... clamatur), les cadences accentuelles (frequéntius agitántur: cursus velox; servíri clamátur : cursus planus) démontrent la maitrise parfaite de la rhétorique du blâme chez Aelred et sa volonté de marquer l'esprit du lecteur par ce paradoxe. La voix passive n'est pas anodine ici : elle manifeste la distance prise vis-à-vis de ce discours tenu, aux dires d'Aelred, par ceux qui défendent la polyphonie : plus le chant est spectaculaire, plus la dévotion serait grande.

Cette volubilité excessive, fruit d'un art perverti (artificiosa), confère donc au chant une portée mimétique (imitari) qui n'a pas sa place dans un lieu de prière. Aussi les fidèles deviennent-ils des spectateurs, ébahis devant tant de débauche visuelle et auditive :

Stans interea vulgus sonitum follium, crepitum cymbalorum, harmoniam fistularum tremens attonitus que miratur; sed lascivas cantantium gesticulationes, meretricias vocum alternationes et infractiones non sine cachinno risu que intuetur, ut eos non ad oratorium, sed ad theatrum, nec ad orandum, sed ad spectandum aestimes convenisse ${ }^{41}$.

Aelred poursuit la description du spectacle offert par les chanteurs en insistant sur son caractère obscène (lascivas, meretricias). L'ouïe (sonitum, crepitum, harmoniam, vocumalternationes et infractiones) et la vue (miratur, intuetur) sont convoquées et provoquent les éclats de rire (cachinno risu). Or le fou rire fait l'objet de critiques récurrentes depuis l'Antiquité. Dans la Rhétorique à Hérennius,

40. Voir Cicéron, L'orateur, 198 ; Quintilien, Institution Oratoire, IX, 4, 54-55.

41. Aelred de Rievaulx, De Speculo Caritatis, II, 23, 68: "Entre-temps, le peuple est là, abasourdi et tremblant, ébahi devant le vacarme des soufflets, le crépitement des cymbales, le concert des flûtes ; mais il ne peut s'empêcher d'éclater de rire en assistant à ces gesticulations lascives des chanteurs, aux brisures de la voix et aux changements de registres dignes de courtisanes, au point qu'on croirait ces gens rassemblés non dans une église mais dans un théâtre, non pour prier mais pour assister à un spectacle». 
il était conseillé à l'orateur d'user de bons mots en respectant les limites de la bienséance : le rire excessif (cachinnatio), distingué du bon rire (risus), risque en effet de déformer (torquere) les mots ${ }^{42}$. La littérature chrétienne, en particulier Augustin, reprenait à son compte cette distinction entre le bon rire et le mauvais rire ${ }^{43}$; il s'agit aussi parfois, comme chez Jérôme, de désapprouver la cocasserie de certaines œuvres lues ou chantées, qui ont un effet déplorable sur la jeunesse qui les pratique ${ }^{44}$. De même, ailleurs chez Aelred, le fou rire est associé à l'ivresse et aux histoires fabuleuses du roi Arthur (fabulae $)^{45}$. Par conséquent, l'évocation du fou rire prend part, de manière évidente, à la rhétorique du blâme déployée par Aelred dans ce chapitre. Ainsi, non seulement la polyphonie pervertit le culte en travestissant les chanteurs, mais elle constitue un obstacle à la prière dans la mesure où l'assemblée des fidèles, loin d'être invitée à se départir du monde extérieur pour entrer dans l'exercice de la méditation, est subjuguée par le spectacle qui se déroule sous ses yeux. La dernière phrase clôt le tableau par une formule frappante et bien rythmée grâce aux antiphrases (non... sed), à l'isosyllabie des homéotéleutes (ad theatrum / ad orandum I ad spectandum) et à la cadence accentuelle (aéstimes convenísse : cursus velox).

Si le blâme exprimé ici par Aelred s'inscrit dans une longue tradition, il porte néanmoins sur un objet précis et contextualisé : la présence d'instruments à l'église et la polyphonie. En outre, cette critique intervient dans un cadre spécifiquement cistercien. Le Miroir de la Charité se fait l'écho des mesures prises par Bernard de Clairvaux pour renforcer la discipline monastique. Ainsi les restrictions apportées par Aelred concernant l'écoute musicale sont-elles le prolongement des limitations apportées à l'usage de l'image matérielle dans le cloître, jugées comme nécessaires face à la dégradation morale et spirituelle qui toucherait la vie monastique. Cet infléchissement spirituel se manifesterait donc dans la musique,

42. Rhétorique à Hérennius, III, 14.

43. Voir notamment Augustin, Sermon 175, PL 38, col. 945 ; Sermon 351, PL 39, col. 1541.

44. Jérôme, Apologie contre Rufin, I, 17 ; Commentaire sur Isaïe, XI, 40, 21.

45. Aelred de Rievaulx, De Speculo Caritatis, II, 6, 306, repris dans le Compendium speculi caritatis, LV, 43. Sur l'association du fou rire et de la fabula, voir également De Institutione Inclusarum, 33 ; De Speculo Caritatis, II, $17,781$. 
à travers la vanité (vanitas) et la volupté (voluptas) dont les chanteurs virtuoses feraient preuve en s'adonnant à la polyphonie.

[...] de his nunc sermo sit, qui sub specie religionis negotium voluptatis obpalliant : qui ea, quae antiqui patres in typis futurorum salubriter exercebant, in usum suae vanitatis usurpant. (...) Sic quod sancti patres instituerunt, ut infirmi excitarentur ad affectum pietatis, in usum assumitur illicitae voluptatis. Non enim sensui praeferendus est sonus, sed sonus cum sensu ad incitamentum maioris affectus plerumque admittendus ${ }^{46}$.

Le rappel de l'auctoritas antique (antiqui patres, sancti patres) permet à Aelred d'attirer l'attention sur le précepte de la Règle bénédictine (chap. 19) selon lequel le chant doit illuminer le sens du texte, et non le diluer sous les vocalises. Ce précepte est repris par Bernard de Clairvaux et constitue la clef de voûte dans la réflexion menée sur le chant monastique dans la célèbre lettre 398 éditée par Claire Maître. Aussi la virtuosité de la polyphonie présente-t-elle deux dangers : l'effacement du sens au profit du seul plaisir musical et la mise en valeur de l'individu qui chante au détriment de la présence divine et angélique, supposée être au cœur du dispositif. En effet, la recherche de la gloire personnelle (in usum suae vanitatis) et la quête d'oreilles complaisantes deviennent la principale motivation de l'exercice du chant.

Aussi le chant n'atteint-il plus l'objectif qui en légitimait l'usage : être un support pour la méditation, ou du moins le développement d'un sentiment de piété pour les fidèles qui ne peuvent s'adonner à une réflexion sur le texte, en raison de capacités intellectuelles ou d'un bagage culturel insuffisants (ut infirmi excitarentur ad affectum pietatis). Ici encore Aelred soigne son expression, comme en témoignent les diverses cadences accentuelles sur les deux vices concernés (cursus planus : vanitátis usúrpant; cursus velox: illícitae voluptátis), les homéotéleutes (praeferendus ; admittendus) ou encore le jeu de répétition des deux concepts essentiels : sonus et

46. Aelred, De Speculo Caritatis, II, 23, 67 et 68: «Parlons maintenant de ceux qui pactisent en secret avec le plaisir sensuel, sous couvert de religion, eux qui usurpent à l'usage de leur vanité ce dont les Pères anciens se servaient comme figures des réalités à venir et en vue de leur salut. [...] Ainsi, ce que les Pères saints ont institué pour que les faibles soient animés de sentiment de piété, est mis au service d'une jouissance illicite ! Car il ne faut pas faire passer le son avant le sens mais joindre généralement l'un à l'autre pour stimuler davantage les pieux sentiments ». 
sensus. Si les adjectifs verbaux témoignent de la visée prescriptive et didactique adoptée dans la fin de l'extrait, l'expression ad affectum pietatis est une réminiscence textuelle qui convoque la réflexion augustinienne et qui tient lieu d'argument d'autorité. Celle-ci est d'ailleurs explicitée dans la suite :

Ait nempe beatissimus Augustinus : movetur animus ad affectum pietatis divino cantico audito : sed si magis sonum quam sensum libido audiendi desideret, improbatur. Et alias : cum me, inquit, magis cantus quam verba delectant, poenaliter me peccasse confiteor, et mallem non audire cantantem ${ }^{47}$.

B. W. Holsinger et E. Dillon rappellent l'importance de la réflexion augustinienne dans la pensée d'Aelred, puisque c'est elle qui tisse la première ce lien entre chant et plaisir charnel ${ }^{48}$. La première citation provient du Contre Julien, où Augustin distingue l'usage réfléchi des sens et la volupté. La seconde citation est extraite du célèbre passage des Confessions où Augustin met en garde contre la tentation de n'écouter que la mélodie, au détriment du texte ${ }^{49}$.

Ces pratiques musicales dénoncées par Aelred détournent la véritable repraesentatio liturgique instaurée par la recitatio. Les conditions pour que l'expérience spirituelle se réalise ne sont pas réunies, car la vision et l'écoute restent prisonnières des sens. Par conséquent, la leçon délivrée par chapitre veut que les émotions ne soient utiles pour la méditation qu'à condition d'être sincèrement ressenties dans un cadre qui précise strictement les frontières de l'expérience collective. En effet, il ne faut pas extérioriser ce que l'on ressent, mais au contraire, entrer en soi. Il s'agit dès lors, comme l'explique D. Boquet, de mettre en place « une dynamique dans laquelle la chair et l'esprit sont orientés dans la même direction, tout

47. Aelred, De Speculo Caritatis, II, 23, 69: «Comme le dit le bienheureux Augustin : “À l'audition d'un cantique divin, l'esprit est porté à un sentiment de piété ; mais il est blâmable si la passion d'entendre lui fait désirer le son plus que le sens !'. Et il dit ailleurs : "Lorsque le chant m'enchante plus que les paroles, je confesse avoir commis un péché qui mérite répréhension, et j'aimerais mieux ne pas entendre chanter" ».

48. B. W. Holsinger, Body, Music and Desire..., p. 73 ; E. Dillon, « Representing Obscene Sound », p. 77.

49. Augustin, Contre Julien, IV , 4, 14, 66 : « Movetur certe animus ad pietatis affectum, divino cantico audito : tamen etiam illic si sonum, non sensum libido audiendi desideret, improbatur». Augustin, Confessions, X, 33: «Tamen cum mihi accidit, ut me amplius cantus quam res, quae canitur, moveat, poenaliter me peccare confiteor et tunc mallem non audire cantantem ». 
en respectant une stricte hiérarchie $\aleph^{50}$. Ainsi, le meilleur spectacle n'est pas celui que l'on a sous les yeux, mais celui qui s'opère à l'abri des regards, dans le for intérieur de chaque individu. Aelred rappelle, sous la forme originale d'un discours à la fois sermon et enseignement, les principes fondamentaux du chant liturgique: c'est la qualité de la dévotion individuelle, aussi bien parmi les exécutants que dans l'auditoire, qui permet à l'expérience collective d'être en conformité avec les préceptes de la Règle bénédictine.

\section{NARRER LES PRÉCEPTES ET CORRIGER LES VICES : LA RHÉTORIQUE EXEMPLAIRE DU GRAND EXORDE}

Dans la cinquième partie du Grand Exorde, Conrad consacre plusieurs chapitres à la psalmodie et aux règles qui doivent dicter le comportement des moines en la matière. Son objectif est pratique : il s'agit de corriger les mauvais penchants des moines, enclins à négliger l'exercice de la psalmodie :

Dignum duximus ad correctionem negligentium pandere qualiter Dominus noster Iesus Christus per se ipsum blasphemam negligentiam cuiusdam clerici corripere dignatus est, quatenus tanto propensius ad emendationem sui negligentium mentes evigilent, quanto sollicitius maiestas corripientis pavido corde pensatur ${ }^{51}$.

Dans cette introduction de chapitre $(\mathrm{V}, 16)$ bien charpentée (dignum duximus... quatenus tanto propensius... quanto sollicitius) et clôturée par une cadence accentuelle (córde pensátur : cursus planus), Conrad dévoile le dispositif auquel il va s'attacher pour dénoncer cette négligence et la corriger (ad correctionem negligentium, blasphemam negligentiam corripere, ad emendationem sui negligentium mentes). Tout d'abord, il entend exposer au grand jour (pandere) le châtiment divin réservé aux moines négligents, en prenant un exemple précis (cuiusdam clerici), susceptible de réveiller les consciences et de faire réagir tout un chacun ( $a d$ emendationem sui). Ce transfert du cas particulier à la prise de conscience collective, puis à l'examen

50. D. Boquet, L'ordre de l'affect..., p. 242.

51. Conrad d'Eberbach, Grand Exorde, V, 16: «Nous croyons donc utile de montrer, pour corriger ce défaut, comment notre Seigneur Jésus Christ daigna reprendre lui-même la négligence blasphématoire d'un clerc; en considérant attentivement, d'un cœur plein d'effroi, la majesté de Celui qui fit cette correction, les tièdes deviendront plus vigilants pour secouer leur propre paresse ». Trad. Jacques Berlioz et al. 
spirituel de l'individu est caractéristique de l'exemplum. En outre, Conrad se présente ici comme un simple intermédiaire entre Dieu et la communauté : sa parole n'est destinée, du moins d'après ce qu'il affirme explicitement, qu'à dévoiler l'action divine et aider les moines à en tirer les conséquences nécessaires. Néanmoins, l'expression pavido corde suppose un travail rhétorique dans la démonstration, visant à susciter la peur chez le lecteur/auditeur. Par conséquent, le dispositif semble bien se fonder sur le pathos, au sens antique du terme, plutôt que sur le logos. Autrement dit, l'amélioration des mœurs est bien appréhendée comme une conséquence directe d'un état émotionnel suscité par l'exemplum, et non comme le résultat d'une réflexion rationnelle sur les inconvénients du péché.

La priorité est donnée à l'exécution des psaumes, en particulier à l'attention et la concentration que cet exercice doit supposer. L'écoute est donc une composante de l'habitus monastique, mais elle n'en est pas la seule. Les récits de Conrad s'attachent moins à la qualité de l'écoute qu'à la concentration du moine, c'est-àdire à la coïncidence entre sa présence physique et sa présence spirituelle, conformément aux préceptes de la Règle bénédictine. Plus précisément, Conrad attire l'attention sur la négligence, qui est un écueil difficile à éviter, en particulier dans le cas d'une psalmodie prolongée lors de vigiles :

Divini servitii cottidianam pensionem cum metu et reverentia summae maiestati nos exhibere psalmista hortatur dicens: Servite Domino in timore. Quia vero imbecillitas cordis humani ad negligentiam ab adolescentia prona facile in spiritalibus studiis tepescit, maxime ubi identitas fastidium generat velut in cursu continuae psalmodiae $[\ldots]^{52}$.

Au début de ce chapitre $(\mathrm{V}, 16)$ est rappelé le précepte que chaque moine doit garder à l'esprit lorsqu'il psalmodie : les termes employés (metus, reverentia et timor) dessinent l'image d'un moine soumis à la majesté divine et qualifient le chant comme la verbalisation de cette soumission. Celle-ci est mise en péril lorsque le moine n'est plus tout à sa tâche et qu'une partie de lui-même échappe à ce dispositif de soumission. Cet égarement est dû à la

52. Conrad d'Eberbach, Grand Exorde, V, 16: «Le Psalmiste nous exhorte à offrir avec crainte et révérence le tribut quotidien du service divin à la Majesté suprême, lorsqu'il dit : Servez le Seigneur dans la crainte (Ps 2,11). Mais la faiblesse du cœur humain le porte à la négligence dès la jeunesse, et il tombe facilement dans la tiédeur au milieu des exercices spirituels, surtout lorsque l'uniformité engendre le dégoût, comme dans le cours d'une psalmodie prolongée ». 
faiblesse intrinsèque de l'homme (imbecillitas cordis humani), mais aussi à la «tyrannie du sommeil » qui subjugue les moines dans la routine instaurée par les veilles prolongées et répétées. La monotonie pousse le moine à exercer sa curiositas, soit qu'il se préoccupe de ce qui se passe autour de lui, soit qu'il rumine des idées et des images sans aucun rapport avec le psaume. Comme Aelred dans le chapitre étudié, c'est cette dualité que dénonce Conrad : sous l'apparence de la prière, se cachent en réalité des pensées futiles qui sont l'œuvre des démons. On retrouve la critique virulente des faux-semblants qui était au cœur du chapitre d'Aelred.

Conrad convoque plusieurs fois l'image des démons dans ses exempla afin de dénoncer les abus de cette négligence. C'est le cas notamment dans un chapitre $(\mathrm{V}, 18)$, où il transmet au lecteur deux récits de démons. Dans le premier, il retrace l'expérience d'un «saint homme » qui appartenait à la maison de Stratfleur en Angleterre. Ce témoignage est censé dévoiler la vérité sur la somnolence lors de l'office, mais aussi sur « les fantasmes et les illusions que les tièdes qui dorment au chœur subissent malgré eux sous l'action des démons $\gg^{53}$. Il révèle que les démons viennent visiter les moines pendant l'office et «insultent les somnolents par des applaudissements très moqueurs et des ricanements » (dormientibus malignissimis applausibus et cachinnis insultare). Les moines endormis fournissent aux démons un spectacle réjouissant, qui suscite les mêmes réactions que celles décrites par Aelred dans son chapitre.

Ainsi le blâme est-il déplacé, du divertissement procuré par la polyphonie obscène à la négligence risible des moines somnolents. Conrad va plus loin encore dans la leçon didactique : outre les applaudissements et les ricanements, les démons s'offrent une récréation en inspirant, dans l'esprit de ces moines négligents, des fantasmagories et des rêves obscènes (phantasmata et turpia somnia). Cette scène est une illustration du constat fait par Conrad, selon lequel la négligence est avant tout liée à la faiblesse naturelle du cœur et aux circonstances. Une ambiguïté est ainsi

53. Conrad d'Eberbach, Grand Exorde, V, 18 : «de phantasiis et illusionibus, quas tepidiores quique etiam in choro dormientes per immissiones daemonum inviti patiuntur $»$. 
maintenue concernant la responsabilité des moines dans ce péché de négligence : les mauvaises pensées s'insinuent dans leur esprit, « malgré eux » (inviti), mais ils devront néanmoins répondre de leurs actes et de leurs pensées devant Dieu, et seront l'objet de châtiments absolument horribles, comme le montre la vision infernale d'un moine au seuil de la mort $(\mathrm{V}, 19)$ : les bourreaux torturaient « un grand nombre d'âmes fixées à un énorme bûcher par des broches et des bâtons pointus » ${ }^{54}$ où " on les faisait rôtir et griller comme des volailles $»^{55}$, ils étendaient les corps sur « des poêles à frire et des plats brûlants pour y recueillir avec grand soin la graisse qui s'écoulait des membres exposés au feu » et «verser à nouveau cette graisse bouillante sur les corps d'où elle s'était écoulée $\aleph^{56}$. Conrad déploie tous ces détails pour imposer une image mentale dans l'esprit de son lecteur/auditeur et susciter l'effroi - en plus, peut-être, de l'appétit. Ainsi, bien que les pensées futiles soient le fruit d'une lassitude bien compréhensible, elles n'en sont pas pour autant pardonnables. La responsabilité du moine est donc reconnue, il lui faut rendre des comptes et il est de son devoir de lutter contre cette propension naturelle à la négligence. Tâche difficile !

Dans le second récit, Conrad reprend un récit provenant de la Vie des Pères, mettant en scène un saint père à qui un esprit malin révèle qu'il assiste fréquemment aux vigiles. Le père en a la confirmation par la suite, grâce à une vision que Conrad interprète comme l'illustration des modalités et des conséquences néfastes de cette négligence :

[...] veniensque ad collectam fratrum vidisset daemones velut Aethiopes per totam ecclesiam discurrentes atque universa, quae fratres orantes et psallentes in cordibus suis versabant, per phantasias in oculis suis figurantes, sicut, postmodum ex confessionibus singulorum patuit, animadversum esse vanas et perversas cogitationes mortiferasque delectationes, quas fratres in hora divini servitii patiuntur, daemonum suggestionibus fieri. Quapropter in spiritu contrito et corde humiliato necesse est exclamare cum psalmista: Ne tradas, Domine, bestiis

54. Conrad d'Eberbach, Grand Exorde, V, 19: «animas scilicet multas verubus et contis acuminatis infixas ».

55. Conrad d'Eberbach, Grand Exorde, V, 19: «ad rogum permaximum in modum anserum torreri et assari ».

56. Conrad d'Eberbach, Grand Exorde, V, 19: «patellas et sartagines ignitas de subtus iacentes ponebant adipemque, qui de ardentibus icentium membris fluebat, diligentissime colligentes eundem rursus bullientem et nimis ferventem super eos, a quibus fluebat, refundebant ». 
animas confitentes tibi et animas pauperum tuorum ne obliviscaris in finem $^{57}$.

Outre l'effroi que cette image mentale des démons est censée susciter, elle met également en lumière «l'opération commando » livrée par la négligence et son armée de démons. L'invasion physique de l'église (per totam ecclesiam discurrentes atque universa) s'accompagne de la production d'images fantasmagoriques (per phantasias in oculis suis figurantes), donnant une forme à ce que Conrad prend le soin de ne pas décrire en détail, mais qui sont bien les pensées vaines et perverses (vanas et perversas cogitationes) qui agitent l'esprit des moines, suscitant un plaisir qui les mènera tout droit en Enfer (mortiferas delectationes). Le lecteur est libre d'imaginer ce qu'il veut, et de verser dans le moule ainsi constitué par Conrad la matière qui lui convient - moyen peut-être pour lui d'effectuer sa propre purification, comme par une sorte de catharsis.

Par ce dispositif de mise en évidence, Conrad fait comprendre les tenants et aboutissants de la négligence, tout en mettant en garde son lecteur : tout moine peut être concerné. Sauver les apparences ne suffit pas, car le péché dissimulé se révèle, tôt ou tard, au grand jour, grâce à l'action des démons ou plus vraisemblablement à la confession (ex confessionibus singulorum), puis par le récit exemplaire lui-même, qui joue ici le rôle d'un relais. Enfin, la citation psalmique traduit un infléchissement énonciatif : Conrad n'accuse plus, mais rappelle le principe désormais clair pour l'esprit de son lecteur, à savoir la nécessaire posture d'humilité et de soumission (necesse est) que le chant est censé signifier aux yeux de tous.

Si Conrad évoque l'exercice de la psalmodie comme une pratique collective, qui nécessite l'attention de chacun, il porte plus précisément son attention sur un excès qui rappelle le texte d'Aelred :

57. Conrad d'Eberbach, Grand Exorde, V, 18: «(...) il vint ensuite à l'assemblée des frères et vit des démons semblables à des Éthiopiens qui couraient çà et là à travers l'église ; ils montrèrent à ses yeux par des images fantastiques tout ce que les frères agitaient dans leur esprit en priant et en chantant, ce qui fut confirmé ensuite par les aveux de chacun d'eux ; et il comprit que toutes les pensées vaines et perverses et les délectations mauvaises qui s'insinuent dans l'esprit des frères au moment de l'office divin ont les démons pour auteurs. C'est pourquoi il nous faut nous exclamer avec le Psalmiste, d'un esprit humilié et d'un cœur contrit : Seigneur, ne livrez pas aux bêtes féroces les âmes de ceux qui vous louent, et n'oubliez pas pour toujours l'âme de vos pauvres $(\mathrm{Ps} 73,19) »$. 
la virtuosité vocale et le péché de vanité. Conrad retranscrit dans un chapitre $(\mathrm{V}, 19)$ un sermon de Bernard de Clairvaux (Sermones super Cantica canticorum, 47, 8) rappelant les principes du chant et enchaîne dans le chapitre suivant $(\mathrm{V}, 20)$ sur l'exemple d'un moine trop vaniteux, qui s'écoute chanter.

Ecce vero dum considero, qualiter vir propheticus miserrimam et nimis superstitiosam quorundam tenaciam reprehendit dicens : Non parcentes vocibus, non fractis et remissis vocibus muliebre quiddam balba de nare sonantes, occurit memoriae terribilis casus, quem referente viro venerabili abbate Morimundi cuidam talium accidisse cognovimus $^{58}$.

Le début de ce chapitre $(\mathrm{V}, 20)$ respecte le dispositif exemplaire (rappel de la source et du principe illustré), mais permet également à Conrad d'attirer l'attention du lecteur sur un passage spécifique du sermon de Bernard transcrit dans le chapitre précédent, qui est l'une des rares indications techniques sur l'usage de la voix dans le milieu cistercien. Comme j'ai eu l'occasion de le souligner ailleurs, l'évocation de la technique vocale s'accompagne ici des recommandations éthiques déjà présentes dans la Règle bénédictine, selon lesquelles le moine doit avant tout préserver sa virilité. On retrouve ici l'un des points essentiels de la démonstration d'Aelred.

La qualification de cet exemple par Conrad (terribilis casus) attise la curiosité du lecteur: de quoi s'agit-il exactement? D'un moine doté d'une voix extraordinaire, qui épargne son organe quand il s'agit de chanter avec ses frères (les hymnes et les psaumes), mais qui déploie une virtuosité hors du commun dans les versets qu'il est chargé de chanter en soliste :

[...] ut versum responsorii sui non plane in gravibus, sed in acutis vel potius in acutissimis vocem quatiendo tinnulosque modulos flexibilitate vocis formando solita lascivia decantaret. Quo finito, ut, a quo merces ridiculosi cantus speranda esset, intelligeret, tam ipso quam ceteris, qui circa eum erant, videntibus et audientibus apparuit daemunculus in modum pueruli Aethiopis taeter et fuscus, qui manibus impuris plausum faciens et miro modo cachinnans

58. Conrad d'Eberbach, Grand Exorde, V, 20 : « Mais voici qu'en réfléchissant à la manière dont cet homme inspiré de Dieu reprenait l'obstination extrême et bien misérable de certains en disant: N'épargnez pas vos voix, ne chantez pas mollement et faiblement comme des femmes, avec des voix nasillardes, il me revient en mémoire une chose horrible arrivée à l'un de ceux qui chantaient de cette manière. Le fait m'a été raconté par le vénérable abbé de Morimond ». 
exclamavit dicens : $\mathrm{O}$, o, bene, valde bene cantavit, optime cantatum est ! 59

Dans cet extrait, l'apparition du démon ne vise pas comme précédemment à inspirer l'effroi du lecteur/auditeur, mais bien plutôt à susciter la moquerie. Plusieurs éléments textuels le suggèrent: la multiplication des suffixes diminutifs (tinnulos, modulos, daemunculus, pueruli) ou bien encore le jugement porté par l'instance énonciative sur le chant, qualifié de ridicule (ridiculosi cantus) en raison de ses notes aiguës ou plutôt suraiguës (acutis vel potius in acutissimis vocem), qui corrompent la virilité du moine et dont le caractère lascif (lascivia) rappelle immédiatement «ces gesticulations lascives des chanteurs, [ces] brisures de la voix et [ces] changements de registres dignes de courtisanes » évoqués par Aelred dans son chapitre.

Mais ici encore, l'intervention des démons permet à Conrad d'aller plus loin dans sa démarche pédagogique. La honte que le moine prétentieux subit est publique, tous les moines rassemblés autour de lui en sont témoins (tam ipso quam ceteris, qui circa eum erant, videntibus et audientibus apparuit...). S'ajoute à ce public le lecteur/auditeur du Grand Exorde, qui assiste lui aussi, à travers l'énoncé exemplaire, au mirum. La réaction du démon est la même que dans le chapitre V, 18 : des applaudissements impurs (manibus impuris plausum faciens) et le ricanement (cachinnans). À cela s'ajoute un discours rapporté au style direct, des félicitations hyperboliques qui provoquent le sarcasme chez le lecteur/auditeur, se félicitant du bon tour joué au moine. Si Conrad cherche moins à provoquer la crainte que la dérision, c'est sans doute parce que le péché de vanité, lié aux qualités vocales exceptionnelles du moine, est moins menaçant pour la communauté, car plus rare, que la négligence qui peut toucher n'importe quel moine.

59. Conrad d'Eberbach, Grand Exorde, V, $20: «(\ldots)$ il chanta le verset de son répons non pas avec simplicité et sur un ton grave, mais sur des notes aiguës ou plutôt suraiguës, avec des trémolos et des vocalises éclatantes et pleines de souplesse, enfin à la manière mondaine qui lui était habituelle. Lorsqu'il eut fini, pour qu'il comprît de qui il devait attendre le salaire de son chant ridicule, un petit démon apparut ; ce frère et ceux qui l'entouraient le virent et l'entendirent également. Il avait l'apparence d'un petit Éthiopien, hideux et noir, qui se mit à applaudir de ses mains impures et à ricaner d'une manière surprenante en s'exclamant : Oh ! Oh ! Il a bien chanté, très bien chanté, c'était admirable!» 


\section{Conclusion}

Dans ce discours du blâme portant sur les mauvaises pratiques de l'écoute et du chant, les deux auteurs s'inscrivent dans la lignée augustinienne, par une mise en garde contre l'attrait que la musique peut représenter au détriment de la méditation ou de tout autre exercice spirituel. Il s'agit alors de mobiliser, en tant que repoussoir, un modèle culturel qui a construit une image de la musique comme un divertissement. En ce sens, nous pouvons parler d'un imaginaire collectif $^{60}$ contre lequel les deux auteurs tentent de lutter, par des dispositifs rhétoriques distincts. Aelred rejette en bloc cette image, parce que précisément elle ne correspond pas au paradigme imposé par Bernard de Clairvaux. Le système antithétique sur lequel est bâti le chapitre XXIII du livre II du Miroir de la Charité présente un tableau antinomique de la mauvaise et de la bonne musique, à la façon des diptyques peignant les délices du Paradis et les châtiments de l'Enfer. Conrad reprend ce système bipolaire, en accentuant le caractère didactique de la démonstration par le biais d'un style plus simple et de procédés narratifs efficaces, notamment dans la mise en scène des démons. En d'autres termes, Aelred s'attache avant tout à construire une identité cistercienne, contre le modèle culturel dominant et séduisant auquel ont été habitués les moines avant leur conversion. Conrad, en revanche, cherche à réaffirmer ces codes identitaires ainsi que leur légitimité, face à des critiques qui émanent autant du milieu cistercien que du dehors, notamment de Cluny.

Cette production cistercienne a bien pour objectif de saper les bases de cet imaginaire collectif, ou plutôt d'en infléchir la valeur axiologique. Il s'agit, en d'autres termes, de remodeler l'image de la musique, de «diaboliser » son caractère divertissant, amusant et spectaculaire. Cette action du langage sur la croyance et les représentations se doublent d'une volonté, très nette chez Aelred

60. Je comprends l'imaginaire collectif au sens que propose Pascal Charaudeau dans «Les stéréotypes, c'est bien, les imaginaires, c'est mieux », Stéréotypage, stéréotypes: fonctionnements, ordinaires et mises en scène, t. 4, Langue(s), Discours, dir. Henri Boyer, Paris, L'Harmattan, 2007, p. 85 (cité par R. Amossy, La représentation de soi..., p. 48) : « les imaginaires sont engendrés par les discours qui circulent dans les groupes sociaux, s'organisant en systèmes de pensée cohérents créateurs de valeurs, jouant le rôle de justification de l'action sociale et se déposant dans la mémoire collective ». 
et Conrad, de modifier, en pratique, le comportement des moines. Mais quel effet a cette rhétorique sur le lecteur/auditeur ? Comment mesurer son efficacité persuasive ? Comme cela a été évoqué à plusieurs reprises, outre les outils rythmiques et stylistiques, les énoncés étudiés mettent en place une stratégie énonciative qui permet de construire un ethos discursif, c'est-à-dire une image de soi que le locuteur parvient à élaborer par le discours. L'efficacité persuasive de cet ethos se mesure à la confiance que le locuteur parvient à mettre en place chez son interlocuteur ${ }^{61}$. Si l'on analyse aujourd'hui cet ethos oratoire cistercien en termes aristotéliciens, il doit rassembler plusieurs qualités pour pouvoir fonctionner: compétence et intelligence (phronesis), honnêteté et sincérité (aretè), bienveillance, solidarité et empathie (eunoia). Or, cette eunoia me semble être le point faible des énoncés analysés, en termes de persuasion et de dispositif rhétorique. L'ethos construit à la fois par Aelred et Conrad se caractérise autant par sa légitimité spirituelle que par son autorité, voire son autoritarisme. Dans ce contexte, le locuteur cherche à imposer plus qu'à construire avec autrui un consensus. Ainsi, l'adhésion ne s'opère pas au travers $\mathrm{du}$ logos, de la rationalité, de l'argumentation, mais s'appuie sur l'ascendant institutionnel et psychologique du locuteur sur son interlocuteur. Cet ascendant relève de la construction discursive d'un cadre axiologique ( il est nécessaire», «il est juste »...), par le biais d'une rhétorique du blâme pleine de véhémence. Cette stratégie répond à une volonté consciente, en tout cas chez Conrad, d'inspirer l'effroi (pavido corde) dans un cadre interactionnel réduit et très hiérarchisé.

formariermarie@gmail.com

61. Aristote, Rhétorique, II, 1378a. Voir R. Amossy, La représentation de soi..., p. 20. 